Ako, S. e146

Aksoy, N. e12

Asselman, M. e103

Assmann, K.J.M. e85

Atahan, I.L. e12

Baliga, M. e17

Baliga, R. e17

Bigler, S.A. e17

Boim, M.A. e94

Cengiz, B. e12

Cengiz, M. e12

Chai, Q. e123

Chai, S. e123

Denhardt, D.T. e66

Denkers, N.D. e154

Dijkman, H.B.P.M. e25

Duim, R.A.J. e103

Dunn, K.W. e7

Fernandez, L. e55

Fukuda, Y. e44
García-Villalba, P. e154

Gerlofs-Nijland, M.E. e25

Goligorsky, M.S. e66

Groenen, P.J.T.A. e25

Gurkaynak, M. e12

Hampel, D.J. e66

Higuchi, M. e146

Hoff, C. e154

Hora, K. e146

Igarashi, P. e1

Ikebe, M. e137

Kamijo, Y. e146

Kluth, D.C. e113

Kowalski, A.J. e66

Koyama, T. e146

Krag, S. e123

Laak, J.A.W.M. van der e85

Leitão, A.A. e94

Leite, C.A. e94

Liu, H. e17
Loeke, N.A.J.M. te e25

Masuda, Y. e44

Mauch, T.J. e154

Miner, J.H. e119, e123

Molitoris, B.A. e7

Mori, T. e44

Mosquera, J. e55

Nakayama, Y. e137

Nelson, R.D. e154

Nonoguchi, H. e137

Nyengaard, J.R. e123

Pozzi, A. e77

Rincón, J. e55

Romanov, V.I. e66

Romero, M. e55

Romijn, J.C. e103

Ryffel, G.U. e35

Sandoval, R.M. e7

Sansome, C. e66
Santos, B.C. e94

Schepers, M.S.J. e103

Schröder, F.H. e103

Shimada, K. e137

Shimizu, A. e44

Son, J.P.H.F. van e25

Tanzawa, K. e137

Tashima, Y. e137

Tomita, K. e137

Verkoelen, C.F. e103

Vural, H. e12

Wetzels, J.F.M. e25

Wilson, H.M. e113

Wittwer, C.T. e154

Wogensen, L. e123

Yamanaka, N. e44

Yildiz, F. e12

Zee, R. van der e25

Zent, R. e77

\title{
Subject Index Vol. 94, 2003
}

Adenovirus e113

Albuminuria e85

Aminopeptidase A e25

Angiotensin receptor e154

Animal cap e 35

AT1A e154

AT1B e154

AT2 e154

ATP depletion e66

Cadherin 16 e1

Capillary repair e44

Catalytic iron e17

CD18 e55

Cell signaling e77

Cisplatin e94

Co-culture e146

Collagen type IV e123

Copper e12

Cre/loxP recombination e1

Crystal retention e103

Cyclosporine e94

Cytochrome P450 e17

- _ inhibitors e17

Cytokine e146

Endothelial cells e44

Endothelin-1 e137

\section{Epitope e25}

Extracellular matrix e77

Fluorescence microscopy e7

Gene e113

Glomerular basement membrane e119

- endothelium e119

- epithelium e119

Glomerulonephritis e113

Glomerulopathy e123

Glomerulus e123

Heme oxygenase e17

Hepatocyte growth factor e146

- nuclear factor 1 el

HNF1 $\beta$ e 35

Hyaluronan e103

Hyperosmotic stress e94

Hypoxia e66

la molecule e55

Inflammation e113

Integrins e77

Intercellular adhesion mole-

$$
\text { cule } 1 \text { e } 55
$$

Intravital optical microscopy e7

Iron e12

Isotypes e123
Kidney e66

-, fetal development e154

-, two-photon microscopy e7

Kidney-specific gene regulation e1

Ksp-cadherin e1

Laminin e123

LightCycler e154

Lim1 e35

LLC-PK1 cells e94

Macrophages e113

Mesangial cell e146

Metal e12

Migration e77

Minimal change nephrotic syndrome e 17

Monoclonal antibodies e25

Monocyte e146

Mouse e25

Nephrolithiasis e103

Nephropathy e12, e123

Nephrosis e55

notch1 e35

Osteopontin e66

Pax2 e35

Pax8 e35
Pericellular matrix e103

Podocyte e85, e119

Progressive glomerulonephritis e44

Proliferating cell nuclear antigen e55

Pronephros e35

Proteinuria natriuresis e137

Puromycin e55

- aminonucleoside e137

Reactive oxygen metabolites e17

Real-time RT-PCR e154

Renal cysts e1

- function e77

- proximal tubular cells e66

Slit diaphragm e85

Total body irradiation e12

Trace element e12

Transforming growth factor- $\beta 1$ e123

Two-photon/multiphoton microscopy e7

Wnt4 e35

WT1 e35

Xenopus e35

\section{KARGER}

Fax +4161306 1234 E-Mail karger@karger.ch www. karger.com
(C) 2003 S. Karger AG, Basel 